\title{
Cardiac Remodeling in Preterm-Born Adults: Long-Term Benefits of Human Milk Consumption in Preterm Neonates
}

\author{
Adam J. Lewandowski
}

$\mathbf{P}$ RETERM BIRTH AFFECTS more than $10 \%$ of infants worldwide and is the leading cause of perinatal morbidity and mortality rates. With appropriate care, most preterm infants survive to adulthood but can suffer from long-term health effects. Data from several animal models suggest that cardiac development may be particularly affected by premature birth. For instance, in a rat model of preterm birth, significant myocardial hypertrophy and increases in interstitial myocardium fibrosis were seen in young (4 weeks) and mature adult (16 weeks) rats compared with controls. These structural changes in the myocardium are associated with heart failure and decreased cardiovascular function, suggesting that preterm birth may increase risk for cardiac disease in adulthood. ${ }^{1}$

The first study demonstrating the impact of preterm birth on left ventricular structure and function in humans found that young adults who were born preterm have significantly increased left ventricular mass, as well as unique left ventricular geometry and reductions in systolic and diastolic function compared with those who were born full term. ${ }^{2}$ Similar differences were seen in right ventricular mass and geometry in young adults born preterm compared with those born at term, with more pronouced reductions in right ventricular systolic function in the preterm-born adults compared with changes seen in the left ventricle. ${ }^{3}$ Differences in left and right ventricular mass between preterm and term infants are seen by 3 months postnatal age, with reduced cardiac volumes in preterm-born neonates and infants. ${ }^{4}$ The early abnormalities in cardiac development seen in preterm infants may translate into increased cardiovascular disease risk later in life. A recent epidemiological study of 2.6 million births between 1987 and 2012 demonstrated a strong association between preterm birth and risk of heart failure in childhood and young adulthood; infants born $<28$ weeks gestation were approximately 17 times more likely to develop heart failure than infants born full term ( $\geq 37$ weeks gestation). ${ }^{5}$ Reduced myocardial reserve in preterm infants may make them less resilient to insults later in life (e.g., myocarditis and hypertension) and more susceptible to heart failure. ${ }^{6}$ This is supported by recent findings that preterm-born young adults have an impaired left ventricular response to physiological stress, suggesting a longterm reduction in myocardial functional reserve. ${ }^{7}$ Early interventions (e.g., pharmacological approaches and nutrition) during the critical period of postnatal cardiac development could be used to improve long-term cardiovascular risk in preterm infants. Human milk provides many health benefits and has been shown to preferentially modify metabolism, immunity, brain development, vascular development, and blood pressure. A follow-up study of preterm infants randomized to receive an exclusive human milk diet $(n=30)$ or an exclusive formula diet $(n=16)$ provided the first evidence of the benefits of breast milk for cardiac function and morphology in adults using cardiovascular magnetic resonance imaging. ${ }^{8}$ Young adults aged 23-28 years who were born prematurely and fed exclusively human milk had increased left and right ventricular end diastolic volumes and stroke volumes adjusted for body size compared with adults who were born preterm and fed exclusively formula. These findings suggest exclusive human milk consumption in preterm infants may improve cardiac morphology and function in adulthood. Furthermore, the exclusive formula group had smaller thoracic volumes and increased pulmonary artery sizes relative to aortic dimensions compared with the exclusive human milk group, suggesting human milk may reduce the risk of pulmonary hypertension in preterm-born individuals.

In summary, preterm birth is associated with altered cardiac shape and function that may increase cardiovascular risk later in life. The postnatal period is a critical time for cardiac development. Consumption of an exclusive human milk diet during this time is beneficial to long-term heart and lung structure and function in preterm infants and may reduce the risk of heart failure and cardiopulmonary disease. Future studies will assess whether human milk can improve myocardial functional reserve capacity and cardiac pump efficiency in preterm-born offspring and whether lactoengineered products (e.g., human milk fortifiers) can impact early life disease risk modification and provide additional cardiac morphological and functional benefits.

\section{References}

1. Bertagnolli M, Dios A, Beland-Bonenfant S, et al. Activation of the cardiac renin-angiotensin system in high oxygenExposed newborn rats: Angiotensin receptor blockade prevents the developmental programming of cardiac dysfunction. Hypertension 2016;67:774-782.

Division of Cardiovascular Medicine, Radcliffe Department of Medicine, University of Oxford, Oxford, United Kingdom. 
2. Lewandowski AJ, Augustine D, Lamata P, et al. Preterm heart in adult life: Cardiovascular magnetic resonance reveals distinct differences in left ventricular mass, geometry, and function. Circulation 2013;127:197-206.

3. Lewandowski AJ, Bradlow WM, Augustine D, et al. Right ventricular systolic dysfunction in young adults born preterm. Circulation 2013;128:713-720.

4. Aye CYL, Lewandowski AJ, Lamata P, et al. Disproportionate cardiac hypertrophy during early postnatal development in infants born preterm. Pediatr Res 2017;82:36-46.

5. Carr H, Cnattingius S, Granath F, et al. Preterm birth and risk of heart failure up to early adulthood. J Am Coll Cardiol 2017;69:2634-2642.

6. Leeson P, Lewandowski AJ. A new risk factor for early heart failure: Preterm birth. J Am Coll Cardiol 2017;69(21):26432645.

7. Huckstep OJ, Williamson W, Telles F, Burchert H, Bertagnolli M, Herdman C, Arnold L, Smillie R, Mohamed A, Boardman H, McCormick K, Neubauer S, Leeson P, Lewandowski AJ.
Physiological stress elicits impaired left ventricular function in preterm-born adults. J Am Coll Cardiol 2018;71:1347-1356.

8. Lewandowski AJ, Lamata P, Francis JM, et al. Breast milk consumption in preterm neonates and cardiac shape in adulthood. Pediatrics 2016;138:e20160050.

Address correspondence to: Adam J. Lewandowski, DPhil Oxford Cardiovascular

Clinical Research Facility

Level 1, John Radcliffe Hospital Division of Cardiovascular Medicine Radcliffe Department of Medicine University of Oxford Oxford OX3 9DU United Kingdom

E-mail: adam.lewandowski@cardiov.ox.ac.uk 